\title{
Relative and almost linking systems
}

\author{
James A. Davis ${ }^{1}$ (D) John B. Polhill ${ }^{2} \cdot$ Ken W. Smith ${ }^{3}$ \\ Received: 28 March 2018 / Accepted: 21 September 2018 / Published online: 15 October 2018 \\ (c) Springer Science+Business Media, LLC, part of Springer Nature 2018
}

\begin{abstract}
Collections of difference sets called linking systems have been used to construct new families of linked systems of symmetric designs. In this paper, we define relative and almost linking systems, collections of difference sets and almost difference sets with very similar linking properties to linking systems. These linking systems have connections to bent sets and vectorial bent functions. We construct examples of relative and almost linking systems using a technical lemma.
\end{abstract}

Keywords Difference sets · Linking systems · Almost difference sets

\section{Introduction}

Recently, difference sets have been used to construct linking systems of designs (see [4, 7] for details), so we provide the following definition.

Definition 1.1 A $k$-subset $D \subset G$ of a group $G$ of order $v$ is a $(v, k, \lambda)$ difference set (DS) if the multiset $\Delta=\left\{d_{1} d_{2}^{-1}: d_{1}, d_{2} \in D\right\}$ contains every nonidentity element exactly $\lambda$ times. The difference set is called reversible if $D^{(-1)}:=\left\{d^{-1}: d \in D\right\}=D$.

James A. Davis: Support was provided by NSA Grant H98230-12-1-0243 and J. Polhill: Funding was provided by National Security Agency (US) Grant H98230-10-1-0216.

$凶 \quad$ James A. Davis

jdavis@ richmond.edu

John B. Polhill

jpolhill@bloomu.edu

Ken W. Smith

kenwsmith@gmail.com

1 Department of Mathematics and Computer Science, University of Richmond, Richmond, VA 23173, USA

2 Department of Mathematics, Computer Science, and Statistics, Bloomsburg University, Bloomsburg, PA 17815, USA

3 Department of Mathematics, Sam Houston State University, Huntsville, TX, USA 
Closely related to difference sets are so-called almost difference sets. Almost difference sets have seen a surge of recent interest due to their practical applications in cryptography (see $[2,5,6,10]$ for details).

Definition 1.2 A $k$-subset $A \subset G$ of a group $G$ of order $v$ is a $(v, k, \lambda, t)$ almost difference set (ADS) if there is a $t$-subset $T \subset G,|T|=t$, so that the multiset $\Delta=\left\{a_{1} a_{2}^{-1}: a_{1}, a_{2} \in A\right\}$ contains every nonidentity element of $T$ exactly $\lambda$ times and every nonidentity element of $G \backslash T$ exactly $\lambda+1$ times. The ADS is called reversible if $A^{(-1)}:=\left\{a^{-1}: a \in A\right\}=A$.

Difference sets and ADSs are often studied in the context of the group ring $\mathbb{Z}[G]$. If we allow the standard abuse of notation by identifying the sets $D, D^{(-1)}$, and $G$ with the group ring elements $D=\sum_{d \in D} d, D^{(-1)}=\sum_{d \in D} d^{-1}$, and $G=\sum_{g \in G} g$ (and we also identify the identity element $1_{G}$ of $G$ with the group ring element $1_{G}$ ), then the subset $D$ is a $(v, k, \lambda)$ DS in $G$ if

$$
D D^{(-1)}=(k-\lambda) 1_{G}+\lambda G .
$$

The subset $D$ is a $(v, k, \lambda, t)$ ADS in $G$ relative to $T$ if

$$
D D^{(-1)}=(k-(\lambda+1)) 1_{G}+(\lambda+1) G-T .
$$

The following definition was introduced in [4].

Definition 1.3 Let $G$ be a finite group of order $v$ and let $\ell \geq 2$. A collection $\left\{D_{i, j}: 0 \leq i, j \leq \ell, i \neq j\right\}$ of $(v, k, \lambda)$ DSs in $G$ is a $(v, k, \lambda ; \ell)$-linking system for $\mu, v \in \mathbb{Z}$ if

- $D_{j, i}=\left(D_{i, j}\right)^{(-1)}$ for all $i \neq j$;

- for all distinct $h, i, j \in\{0, \ldots, \ell\}, D_{h, i} D_{i, j}=\mu D_{h, j}+v\left(G-D_{h, j}\right)$.

We call the linking system reversible if all of the DSs in the collection are reversible.

We note that $\mu=\frac{k(k \pm \sqrt{k-\lambda})}{v} \mp \sqrt{k-\lambda}$ and $v=\frac{k(k \pm \sqrt{k-\lambda})}{v}$ can both be computed from the parameters $v, k$, and $\lambda$. These parameters must be integers, so we can only have linking systems when $v, k$, and $\lambda$ can produce integer values for $\mu$ and $\nu$. This paper considers the question of other linking behaviors that occur even when a linking system is not possible. We also refer to [7] for nonexistence results for linking systems.

The first examples of linking systems were found in [3] in the context of bent sets. A bent set is a set of bent functions with the property that the sum of any two elements of the set is also a bent function. A bent set is called closed if the sum of any two bent functions in the set is also in the set, and a bent set is called a Kerdock set if the size of the set is as large as possible. The examples in [3] are Kerdock sets, and closed bent sets can be constructed by vectorial bent functions (see [9] for background on vectorial bent functions). The examples of linking systems found in [4] can be thought of as generalizations of bent sets since they occur in nonelementary abelian groups. 
We will usually define a linking system by specifying a set $S=\left\{D_{i, 0} \mid 0<i \leq \ell\right\}$ since (i) $D_{0, i}=D_{i, 0}^{(-1)}$ for all $0<i \leq \ell$ and (ii) $D_{i, j}$ can be recovered from $S$ and the equation $D_{i, 0} D_{0, j}=\mu D_{i, j}+v\left(G-D_{i, j}\right)$. We will call a linking system closed if the linking system has the property that for all $0 \leq i, j \leq \ell, i \neq j, D_{i, j}=D_{m, 0}$ for some $1 \leq m \leq \ell$. A closed linking system can be thought of as analogous to a vectorial bent function.

The next definition contains the main objects for the rest of the paper. These are new definitions, and they are related to the linking systems in Definition 1.3.

Definition 1.4 Relative linking system: Let $G$ be a finite group of order $v$ and let $\ell \geq 2$. A collection $\left\{D_{i, j}: 0 \leq i, j \leq \ell, i \neq j\right\}$ of $(v, k, \lambda)$ DSs in $G$ is a $(v, k, \lambda ; \ell+1, c)$-relative linking system for $\mu, v \in \mathbb{Z}$ relative to a subgroup $H$ if

- $D_{j, i}=\left(D_{i, j}\right)^{(-1)}$ for all $i \neq j$;

- for all distinct $h, i, j \in\{0, \ldots, \ell\}, D_{h, i} D_{i, j}=\mu D_{h, j}+v\left(G-D_{h, j}\right)+c H$.

Almost linking system Let $G$ be a finite group of order $v$ and let $\ell \geq 2$. A collection $\left\{D_{i, j}: 0 \leq i, j \leq \ell, i \neq j\right\}$ of $(v, k, \lambda, t)$ ADSs in $G$ is a $(v, k, \lambda, t$; $\ell+1)$-almost linking system for $\mu, v \in \mathbb{Z}$ if

- $D_{j, i}=\left(D_{i, j}\right)^{(-1)}$ for all $i \neq j$;

- for all distinct $h, i, j \in\{0, \ldots, \ell\}, D_{h, i} D_{i, j}=\mu D_{h, j}+v\left(G-D_{h, j}\right)$.

We call the linking system reversible if all of the ADSs in the collection are reversible.

In both cases, we will call the linking system closed if for all $0 \leq i, j \leq \ell, i \neq j$, we have $D_{i, j}=D_{m, 0}$ for some $1 \leq m \leq \ell$. In that case, we will write $D_{m, 0}=D_{m}$.

The following lemma will be used in the constructions in the next section. We delay the proof of this lemma until the final section. If $Q=2^{a}$, $\xi$ is a primitive element of the field $G F(Q), G=G F(Q)^{+}$, and $\oplus$ is addition in $G$, then we have the following result in $\mathbb{Z}[G]$.

Lemma 1.5 If $0 \leq j<k \leq Q-2$, then $\sum_{i=0}^{Q-2} \sum_{n=0, n \neq i}^{Q-2} \xi^{i+j} \oplus \xi^{n+k}=(Q-$ 3) $G+2 \cdot 0_{G}$.

\section{Relative linking system example}

Let $H=E A(125)$, the elementary abelian group of order 125 , and let $H_{i}, 1 \leq i \leq 31$, be the hyperplanes of $H$ (subgroups of order 25). We will need Lemma 1.5, so we define $G:=G F(Q)^{+}$for $Q=32$, and let $\xi$ be a primitive element of $G F(32)$. Define the following subsets of $G \times H$ :

$$
D_{j}=\sum_{i=1}^{31} \xi^{i+j} H_{i}, \quad 1 \leq j \leq 31 .
$$

McFarland [8] showed that all of the $D_{j}$ are reversible $(4000,775,150)$ DSs in $G \times H$. The following theorem shows that the DSs above form a closed relative linking system relative to $\{0\} \times H$. 
Theorem 2.1 The collection $\left\{D_{j}: 1 \leq j \leq 31\right\}$ defined above is a closed reversible $(4000,775,150 ; 32,10)$-relative linking system of $D S$ s in $G \times H$ relative to $\{0\} \times H$.

Proof We need to compute the product of two distinct elements of this set, so assume $j \neq k$.

$$
\begin{aligned}
D_{j} D_{k} & =\sum_{i=0}^{30} \xi^{i+j} H_{i} \sum_{n=0}^{30} \xi^{n+k} H_{n} \\
& =5 \sum_{i=0}^{30} \sum_{n=0, n \neq i}^{30}\left(\xi^{i+j} \oplus \xi^{n+k}\right) H+25 \sum_{i=0}^{30} \xi^{i}\left(\xi^{j} \oplus \xi^{k}\right) H_{i} \\
& =145(G \times H)+10(\{0\} \times H)+25 D_{m} .
\end{aligned}
$$

The first term in the second line is the computation for $i \neq n$, and it uses the fact that $H_{i} H_{n}=5 H$. The second term in the second line is the computation for $i=n$, and it uses the fact that $H_{i} H_{i}=25 H_{i}$. Lemma 1.5 implies that the first term is $5\left(29 G+2 \cdot 0_{G}\right) H=145 G \times H+10 H$. If we replace $\xi^{i}\left(\xi^{j} \oplus \xi^{k}\right)$ with $\xi^{i+m}$ for the appropriate $m$ satisfying $\xi^{j} \oplus \xi^{k}=\xi^{m}$, then we see that the second term is $D_{m}$.

Two quick remarks. First, we may be able to get even more linking systems of McFarland DSs if we do not require reversible DSs for relative linking systems. Second, the number of DSs in this closed relative linking system, $\ell=31$, seems quite large, which is good when we consider the analogy to bent sets.

\section{Almost linking systems}

Consider the groups $H=\mathbb{Z}_{p}^{2}, G=G F(p+1)^{+}$for $p$ a prime satisfying $Q=$ $p+1=2^{a}$ for some $a$ (so $p$ is a Mersenne prime and $Q$ is from Lemma 1.5). Let $H_{i}, 0 \leq i \leq p$ be the hyperplanes of $H$ (subgroups of order $p$ ) and let $\xi$ be a primitive element of $G F(p+1)$. Define

$$
D_{j}=H_{0}+\sum_{i=1}^{p} \xi^{i+j} H_{i}, \quad 1 \leq j \leq p .
$$

Arasu et al. [1] showed that these sets are $\left(p^{2}(p+1), p(p+1), p, p(p+1)\right)$ reversible ADSs in $G \times H$. We claim that $\left\{D_{j} \mid 1 \leq j \leq p\right\}$ form a closed reversible almost linking system.

Theorem 3.1 The collection $\left\{D_{j}: 1 \leq j \leq p\right\}$ defined above is a closed reversible $\left(p^{2}(p+1), p(p+1), p, p(p+1), p\right)$-almost linking system in $G \times H$.

Proof We have a similar proof as that in Theorem 2.1. For $j \neq k$, we compute 


$$
\begin{aligned}
D_{j} D_{k} & =\left(H_{0}+\sum_{i=1}^{p} \xi^{i+j} H_{i}\right)\left(H_{0}+\sum_{n=1}^{p} \xi^{n+k} H_{n}\right) \\
& =p H_{0}+\sum_{n=1}^{p} \xi^{n+k} H+\sum_{i=1}^{p} \xi^{i+j} H+\sum_{i=1}^{p} \sum_{n=1}^{p}\left(\xi^{i+j} \oplus \xi^{n+k}\right) H_{i} H_{n} .
\end{aligned}
$$

We used the fact that $H_{i} H_{n}=H$ when $i \neq n$ and $H_{i} H_{i}=p H_{i}$. The sums $\sum_{i=1}^{p} \xi^{i+j} H=G \times H-(\{0\} \times H)$ and $\sum_{n=1}^{p} \xi^{n+k} H=G \times H-(\{0\} \times H)$ since each of those sums is missing the subgroup $(\{0\} \times H)$. The final sum $\sum_{i=1}^{p} \sum_{n=1}^{p}\left(\xi^{i+j} \oplus\right.$ $\left.\xi^{n+k}\right) H_{i} H_{n}$ can be broken up into the cases where $i \neq n$ and $i=n$.

$$
\sum_{i=1}^{p} \sum_{n=1, n \neq i}^{p}\left(\xi^{i+j} \oplus \xi^{n+k}\right) H_{i} H_{n}=\sum_{i=1}^{p} \sum_{n=1, n \neq i}^{p}\left(\xi^{i+j} \oplus \xi^{n+k}\right) H
$$

By Lemma 1.5, we get

$$
\left((p-2) G+2 \cdot 0_{G}\right) H=(p-2) G \times H+2(\{0\} \times H) .
$$

Lastly, we do the sum where $i=n$, which is $p \sum_{i=1}^{p} \xi^{i}\left(\xi^{j} \oplus \xi^{k}\right) H_{i}=$ $p \sum_{i=1}^{p} \xi^{i+m} H_{i}=p\left(D_{m}-H_{0}\right)$. (As in Lemma 1.5, there is an $m$ so that $\xi^{j} \oplus \xi^{k}=\xi^{m}$ since $\xi^{j} \oplus \xi^{k}$ is nonzero.)

Putting this all together we get

$$
\begin{aligned}
D_{j} D_{k} & =p H_{0}+2(G \times H-(\{0\} \times H))+(p-2) G \times H+2(\{0\} \times H)+p D_{m}-p H_{0} \\
& =p\left(G \times H-D_{m}\right)+2 p D_{m} .
\end{aligned}
$$

\section{Proof of Lemma 1.5}

We start by considering the group ring sum over all terms of the form $\xi^{i+j} \oplus \xi^{n+k}$ without restricting to $n \neq i$.

$$
\begin{aligned}
\sum_{i=0}^{Q-2} \sum_{n=0}^{Q-2} \xi^{i+j} \oplus \xi^{n+k} & =\sum_{i=0}^{Q-2}\left(G-\xi^{i+j}\right) \\
& =(Q-1) G-\left(G-0_{G}\right) \\
& =(Q-2) G+0_{G}
\end{aligned}
$$

The first equality follows from the observation that the elements $\xi^{n+k}$ will range over all nonzero elements of the field as $n$ ranges from 0 to $Q-2$, and hence the field sum $\xi^{i+j} \oplus \xi^{n+k}$ will take on all field element values except $\xi^{i+j}$. The second equality follows from a similar observation that the elements $\xi^{i+j}$ will range over all nonzero values as $i$ ranges from 0 to $Q-2$. 
The claim we are trying to prove only includes the terms where $n \neq i$, so we need to subtract off the terms where $n=i$. We compute that sum in the following.

$$
\begin{aligned}
\sum_{i=0}^{Q-2} \xi^{i+j} \oplus \xi^{i+k} & =\sum_{i=0}^{Q-2} \xi^{i}\left(\xi^{j} \oplus \xi^{k}\right) \\
& =\sum_{i=0}^{Q-2} \xi^{i} \xi^{m} \\
& =G-0_{G} .
\end{aligned}
$$

The second equality follows from the fact that $\xi^{j} \oplus \xi^{k}=\xi^{m}$ for some $0 \leq m \leq$ $Q-2$ since all nonzero elements of the field (of which $\xi^{j} \oplus \xi^{k}$ is nonzero since $j<k$ : this is where we need $Q$ to be a power of 2 so that every element is an involution in the additive group. We may be able to modify this argument for other prime powers) can be expressed as a power of $\xi$.

Combining the two equations (subtracting the second equation from the first) yields the result.

\section{References}

1. Arasu, K.T., Jungnickel, D., Pott, A.: Divisible difference sets with multiplier-1. J. Algebra 133(1), 35-62 (1990)

2. Arasu, K.T., Ding, C., Helleseth, T., Kumar, P.V., Martinsen, H.M.: Almost difference sets and their sequences with optimal autocorrelation. IEEE Trans. Inf. Theory 47(7), 2934-2943 (2001)

3. Cameron, P.J., Seidel, J.J.: Quadratic forms over GF(2). Proc. Kon. Neder. Akad. Wetensch. (A) 76, 1-8 (1973)

4. Davis, J.A., Martin, W.J., Polhill, J.B.: Linking systems in nonelementary abelian groups. J. Combin. Theory (A) 123, 92-103 (2014)

5. Ding, C.: Codes from Difference Sets. World Scientific, Singapore (2015)

6. Ding, C., Pott, A., Wang, Q.: Constructions of almost difference sets from finite fields. Des. Codes Crypt. 72(3), 581-592 (2014)

7. Jedwab, J., Li, S., Simon, S.: Linking systems of difference sets. arXiv:1708.04405 (2017)

8. McFarland, R.L.: A family of difference sets in non-cyclic groups. J. Combin. Theory (A) 15(1), 1-10 (1973)

9. Mesnager, S.: Bent Functions: Fundamentals and Results. Springer, Berlin (2016)

10. Nowak, K.: A survey on almost difference sets. arXiv:1409.0114 (2014) 